$(\mathrm{p}<0.001)$ for presence of FFICCs. A statistically significant (SS) decrease in overall survival (OS) was noted among patients with FFICCs $(\mathrm{p}=0.045)$, however progression free survival (PFS) was not found to be SS.

Conclusion* The presence of FFICCs may provide important prognostic information specifically with regard to determining adjuvant treatment in those with positive cytology and/or high risk histologies. In addition, the higher incidence among robotic hysterectomies may warrant a closer look at mode of uterine manipulation. Lack of difference in PFS with statistical significance in OS suggests either a difference in the pattern of recurrence or possibly in postoperative therapy.

\section{UTERINE CARCINOSARCOMA: A MULTICENTRE REVIEW OF TREATMENT AND OUTCOMES OVER 26 YEARS}

${ }^{1} \mathrm{C}$ Yim* ${ }^{*}{ }^{2} \mathrm{SE}$ Yao, ${ }^{3} \mathrm{~J}$ Phung, ${ }^{4} \mathrm{M}$ Davis-Tuck, ${ }^{2} \mathrm{~T}$ Manolitsas, ${ }^{2} \mathrm{~J}$ Mcneilage, ${ }^{2} \mathrm{~K}$ Reid, ${ }^{5} \mathrm{O}$ Mcnally, ${ }^{6} \mathrm{R}$ Rome, ${ }^{2} \mathrm{~T}$ Jobling. ${ }^{1}$ Royal Hospital for Women, Gynaecological Cancer Centre, Randwick, Australia; ${ }^{2}$ Monash Health - Moorabbin Hospital, Gynaeoncology Unit, Bentleigh East, Australia; ${ }^{3}$ University of Newcastle, School of Medicine and Public Health, Callaghan, Australia; ${ }^{4}$ Hudson Institute of Medical Research, Clayton, Australia; ${ }^{5}$ The Royal Women's Hospital, Oncology and Dysplasia Service, Parkville, Australia; ${ }^{6}$ Epworth Freemasons Hospital, East Melbourne, Australia

\subsection{6/ijgc-2021-ESGO.184}

Introduction/Background* Uterine carcinosarcoma (UCS) is a rare and aggressive subtype of endometrial cancer with carcinomatous and sarcomatous elements. It comprises less than $5 \%$ of all uterine malignancies but carries the worst prognosis. The rarity of this disease and its inclusion in heterogenous groups with other rare uterine neoplasms has resulted in limited and unclear evidence to guide best practice. Our aim was to review the characteristics, treatment and outcomes of UCS cases across two public gynaeoncology units and five private gynaeoncology practices in Melbourne over 26 years.

Methodology A retrospective observational study was conducted where UCS cases were identified from hysterectomy pathology records between 1994 and 2020 inclusive. Patient demographics, treatment details, recurrence and survival data were extracted from patient records. Ethics exemptions were obtained from each of the involved institutions.

Result(s)* 208 cases of UCS were identified. The overall recurrence rate was $26.0 \%$ and the overall death rate was $60.1 \%$. Increasing age at diagnosis was associated with an increased risk of death (adjusted OR 1.04, 95\% CI 1.01-1.08, $\mathrm{p}=0.019)$. Risk of death was highest in Stage III disease (adjusted OR 4.37, 95\% CI 1.67-11.40). Recurrence was a strong determinant of death, with an adjusted OR of 7.58 $(p<0.001)$. These predictors of survival were independent of modality of adjuvant therapy.

Conclusion* This is the largest Australian series of homogenous UCS cases to date, and one of the largest cohorts worldwide. This adds important information to the existing body of evidence regarding UCS.

\section{TVS VS MRI FOR ASSESSING MYOMETRIAL INFILTRATION IN ENDOMETRIOID LOW GRADE ENDOMETRIAL CANCER: A PROSPECTIVE STUDY}

'BR Gastón Moreno*, ${ }^{2} \mathrm{~L}$ Alcazar, 'JC Muruzábal Torquemada, 'S Lapeña Calavia, ${ }^{1}$ Al Modroño Blanco, ${ }^{1} \mathrm{R}$ Guarch Troyas, ${ }^{1} \mathrm{CM}$ Tauste Rubio. ${ }^{1}$ Complejo Hospitalario de Navarra, Obstetrics and Gynecology, Pamplona, Spain; ${ }^{2}$ Clinica Universidad de Navarra, Obstetrics and Gynecology, Pamplona, Spain

\subsection{6/ijgc-2021-ESGO.185}

Introduction/Background* Determining the degree of myometrial infiltration in endometrial cancer allows establishing the best therapeutic approach for each patient as it is an important factor in predicting nodal metastases.

Few prospective studies comparing the diagnostic performance of transvaginal ultrasound (TVS) and magnetic resonance imaging (MRI) in the preoperative local staging of endometrial carcinoma have been reported. In fact, a recent meta-analysis has shown that both techniques have similar diagnostic accuracy. However, to the best of our knowledge, there has been no prospective comparison of the diagnostic performance of TVS and MRI in the same group of patients with low-grade endometrial cancer.

The aim of this study was to assess and compare the diagnostic performance of transvaginal ultrasound, magnetic resonance imaging (MRI) and intraoperative pathological study for detecting deep myometrial infiltration in patients with a preoperative endometrial biopsy result of low-grade (G1/G2) endometrioid endometrial cancer.

Methodology Observational prospective study performed at a single tertiary care centre from 2016 to 2020, comprising 156 consecutive patients diagnosed by endometrial sampling as having an endometrioid grade 1 /grade 2 endometrial cancer. TVS and MRI were performed prior to surgical staging for assessing MI, which was estimated using subjective examiner's impression and Karlsson's method for both TVS and MRI. During surgery, intraoperative assessment of MI was also performed. Definitive pathological study considered as reference standard. Diagnostic accuracy for ultrasound, MRI and intraoperative biopsy was estimated and compared.

Result(s)* Sensitivity and specificity of TVS for detecting deep MI were $75 \%$ and $73.5 \%$ for subjective impression and $65 \%$ and $70 \%$ for Karlsson method, respectively $(p=0.54)$. Sensitivity and specificity of MRI for detecting deep MI were $80 \%$ and $87 \%$ for subjective impression and $70 \%$ and $71.3 \%$ for Karlsson method. MRI subjective impression showed a significant better specificity than MRI Karlsson method $(p=0.03)$. MRI showed better specificity than TVS when subjective impression was considered $(\mathrm{p}<0.05)$, but not for Karlsson method. Sensitivity and specificity of intraoperative were $75 \%$ and 97\%, respectively. Intraoperative biopsy showed better specificity than ultrasound and MRI either using examiner's impression or Karlsson method ( $\mathrm{p}<0.05$ )

Conclusion* MRI revealed a significant higher specificity than TVS when assessing deep myometrial infiltration. However, the intraoperative biopsy offers a significant better diagnostic accuracy than preoperative imaging techniques 